\title{
Horizons in Nutritional Science
}

\section{Folic acid supplementation in pregnancy: are there devils in the detail?}

\author{
Graham C. Burdge ${ }^{1 *}$ and Karen A. Lillycrop ${ }^{2}$ \\ ${ }^{1}$ Academic Unit of Human Development and Health, Faculty of Medicine, Institute of Developmental Sciences Building \\ (MP887), Southampton General Hospital, University of Southampton, Tremona Road, Southampton SO16 6YD, UK \\ ${ }^{2}$ Faculty of Natural and Environmental Sciences, Institute of Developmental Sciences Building, Southampton General \\ Hospital, University of Southampton, Tremona Road, Southampton SO16 6YD, UK
}

(Submitted 25 July 2012 - Final revision received 26 July 2012 - Accepted 27 July 2012 - First published online 9 October 2012)

\begin{abstract}
Maternal folic acid (FA) supplementation is well recognised to protect against neural tube defects. Folate is a critical cofactor in one-carbon metabolism involved in the epigenetic regulation of transcription that underpins development. Thus, it is possible that maternal FA supplementation may have additional, unforeseen persistent effects in the offspring. This is supported by the modification by maternal supplementation with one-carbon donors and FA of the epigenetic regulation of offspring phenotype in mutant mice. The present article reviews studies in human subjects and experimental animals of the effect of maternal FA intake and phenotypic outcomes in the offspring. Maternal FA intake was associated with a short-term increased incidence of allergy-related respiratory impairment in children and multigenerational respiratory impairment in rats. Higher maternal folate status during pregnancy was associated positively with insulin resistance in 6-year-olds. In rats, maternal FA supplementation modified hepatic metabolism and vascular function through altered transcription, in some cases underpinned by epigenetic changes. FA supplementation in pregnant rats increased mammary tumorigenesis, but decreased colorectal cancer in the offspring. Maternal FA supplementation decreased a range of congenital cardiac defects in children. These findings support the view that maternal FA supplementation induces persistent changes in a number of phenotypic outcomes in the offspring. However, the number of studies is limited and insufficient to indicate a need to change current recommendations for FA intake in pregnancy. Nevertheless, such effects should be investigated thoroughly in order to support firm conclusions about the risk of unanticipated long-term negative effects of maternal FA supplementation in humans.
\end{abstract}

Key words: Folic acid: Epigenetics: Disease risk

Folates are a group of B-vitamins differing in oxidation state, addition of one-carbon moieties and length of the polyglutamate chain; they are found predominantly in fruits and vegetables. Folic acid (FA) is the synthetic oxidised monoglutamyl form of folate that is widely used in vitamin supplements and in the fortification of foods. Within cells, folates act as cofactors in numerous reactions related to growth and repair requiring the transfer of one-carbon units, principally methyl or formyl groups. These include purine and pyrimidine biosynthesis, and via the homocysteine-methionine methylation cycle, phosphatidylcholine biosynthesis by phosphatidylethanolamine $N$-methylation and the epigenetic control of gene transcription by DNA and histone methylation.

Much of the interest in FA lies in the impact of dietary supplementation and fortification strategies in reducing the incidence of neural tube defects (NTD) ${ }^{(1)}$. NTD, the most common congenital malformation, are caused by the failure of the neural tube to close during embryogenesis between post-conception days 21 and 28, resulting in fatal anacephally or spina bifida with varying levels of disability. The estimated global incidence of NTD is about $>300000$ new cases per year with an estimated 41000 deaths ${ }^{(2)}$. To date, the precise cause of NTD is not known, but is thought to represent the interaction between genetic disposition and environmental factors, including nutrition ${ }^{(1)}$.

FA was first proposed as a means of preventing NTD in $1964^{(3)}$. In four out of five subsequent case-control studies of FA supplementation together with other vitamin supplements and in one non-randomised prospective cohort study in women who did not have a previous NTD-affected

Abbreviations: $\mathrm{A}^{\mathrm{VY} / \mathrm{a}}$, agouti 'Viable Yellow'; FA, folic acid; NTD, neural tube defect; PR, protein restricted.

*Corresponding author: Dr G. C. Burdge, fax +44 238079 5255, email g.c.burdge@soton.ac.uk 
pregnancy, reductions of $35-75 \%$ in the incidence of NTD have been reported ${ }^{(4-7)}$. Similar positive outcomes have also been reported in non-randomised and randomised recurrence trials ${ }^{(8-12)}$. Based on this evidence, a number of countries have introduced recommendations for FA supplementation before and during the first trimester at $400 \mu \mathrm{g} / \mathrm{d}$ increasing up to $5 \mathrm{mg} / \mathrm{d}$ for women with a previous NTD-affected pregnancy. Unfortunately, the effectiveness of recommendations for FA supplementation in reducing the incidence of NTD has been less than that indicated by the trials ${ }^{(13)}$. This reflects the findings that, for example, about $50 \%$ of pregnancies in the USA are unplanned ${ }^{(14)}$ and less than $25 \%$ of women follow the health guidelines ${ }^{(15,16)}$. Consequently, fifty-three countries have regulations for mandatory fortification of flour with FA (typically between 40 and $220 \mu \mathrm{g} / 100 \mathrm{~g}$ ), although these are not implemented in all nations ${ }^{(17)}$. Implementation of FA fortification has, in general, led to significant increases in folate status and reductions in NTD incidence, although the effectiveness appears to be greatest in populations with the highest incidence of NTD ${ }^{(17)}$.

FA is generally considered safe, with a tolerable upper intake level of $1 \mathrm{mg} / \mathrm{d}$ and a lowest observed adverse effect level of $5 \mathrm{mg} / \mathrm{d}^{(18)}$. Even intakes of $15-100 \mathrm{mg} / \mathrm{d}$ are only associated with limited direct toxicity ${ }^{(19-22)}$. Thus, using FA supplements during pregnancy is viewed as a safe and potentially effective means of reducing the incidence of NTD and the associated cost in terms of human suffering and economic burden. Folate is an essential cofactor for the synthesis of purines and thymidylate, and thus plays an important role in DNA synthesis and replication. Folate also plays a central role in epigenetic processes that regulate the transcription of all genes and, in turn, the phenotype of an organism. Folate restriction and supplementation modify epigenetic marks, in particular DNA methylation, by a mechanism that involves DNA methyltransferase activity but has not been described completely. This raises a potential concern that FA supplementation during pregnancy to reduce the risk of NTD may induce unforeseen changes in the methylome and phenotype of the offspring. The purpose of the present review is to draw on studies in human subjects and in animal models to evaluate the effect of FA supplementation during pregnancy on phenotypic and molecular outcomes in the offspring and to indicate gaps in current knowledge.

\section{Folate and the epigenetic regulation of transcription}

Epigenetic processes (DNA methylation, covalent modification of histones and non-coding RNA) regulate gene expression over time scales ranging between minutes for some genes involved in acute energy production ${ }^{(23)}$ to the entire life course for imprinted genes and genes involved in maintaining cell differentiation $^{(24)}$. Folate is a critical cofactor for the provision of methyl groups via the homocysteine-methionine methylation cycle for induction and maintenance of both DNA and histone methylation. It is likely, therefore, that any effects of maternal FA supplementation on the phenotype of the offspring would be mediated via an epigenetic mechanism. There is some evidence which shows that variations in dietary FA can modify the activities of DNA methyltransferases and histone methyltransferases. DNA methylation and histone modification are processes that are intimately linked and while a number of studies have shown that DNA methylation can direct histone modification ${ }^{(25)}$, other studies have shown that histone modifications, particularly histone methylation, precede DNA methylation ${ }^{(26)}$. To date, studies have focused almost exclusively on the effect of FA supplementation on the DNA methylome, although many of the effects of FA may be initiated through the altered activities of histone methyl transferases and histone methylation. Such studies in animal models, which are summarised below, have shown a causal link between maternal FA supplementation, and the epigenome and phenotype of the offspring. However, at present, only one study has reported the effects of maternal FA supplementation on epigenetic marks in children. Fryer et $a{ }^{(27)}$ have shown using an unbiased analysis of 27000 CpG loci in twelve pregnancies, of which eight out of twelve women took FA supplements, that methylation of specific CpG dinucleotides within LINE-1 elements (a surrogate for genome-wide methylation) was associated with birth weight and cord plasma homocysteine concentration, although the direction of association differed between CpG loci. Of the 540 such loci, sixteen were associated with all three outcomes. These findings support a causal association between maternal FA supplementation, epigenetic regulation and offspring phenotype, although the mechanism that links variation in DNA methylation and birth weight was not investigated. Furthermore, since variation in birth weight has been associated with the differential risk of non-communicable diseases ${ }^{(28)}$, these findings point towards a potential association between maternal FA intake and vulnerability to future disease.

\section{Maternal methyl donor supplementation in mutant mice}

There are two mouse models that provide clear examples of the effect of maternal methyl donor intake on the phenotype of the offspring. Agouti 'Viable Yellow' $\left(\mathrm{A}^{\mathrm{VY} / \mathrm{a}}\right)$ and Axin(Fu) bear naturally occurring mutations involving transposons where DNA methylation controls the activity of cryptic promoters linked to the mutant phenotype. $\mathrm{A}^{\mathrm{VY} / \mathrm{a}}$ in which the proximal intracisternal A particle long terminal repeat is hypomethylated have yellow coat colour and an increased tendency towards obesity, some cancers and shorter lifespan compared with black-coated $\mathrm{A}^{\mathrm{VY} / \mathrm{a}}$ mice in which the intracisternal A particle promoter is hypermethylated. Feeding pregnant dams a diet containing increased amounts of methyl donors and FA induces the switching of the offspring phenotype from the yellow- to black-coated phenotype ${ }^{(29)}$. Similarly, supplementation of the diet of dams with methyl donors switches the phenotype of the offspring carrying the Axin(Fu) epiallele, reducing the incidence of tail kinking by half $^{(30)}$. Although these models represent extreme cases, they point towards the potential for vulnerability due to the rather surprising level of plasticity in the epigenome to nutritional inputs by which methyl donors and cofactors, including FA, in the maternal diet induce persistent changes in the phenotype of the offspring. 


\section{Effect of maternal folic acid supplementation on offspring allergy disease}

A number of studies have investigated the extent to which maternal FA supplementation is associated with the risk of respiratory disease risk or allergy in childhood. Haberg et al. ${ }^{(31)}$ found using self-reported questionnaires in 32077 subjects that maternal FA supplementation during the first trimester significantly increased the relative risk of wheeze by $6 \%$, of lower respiratory tract infection by $9 \%$ and of hospitalisation due to lower respiratory tract infection by $24 \%$ up to 18 months of age. Maternal FA supplementation between 30 and 34 weeks of gestation was associated with a $26 \%$ increase in the relative risk of physician-diagnosed asthma at 3.5 years among 490 children, but not at 5 years, while there was no association with FA supplementation before 16 weeks of gestation $^{(32)}$. In a study of 628 pregnancies, a supplemental intake of $>500 \mu \mathrm{g} \mathrm{FA} / \mathrm{d}$ was associated with an $85 \%$ greater risk of allergic disease, mainly eczema, than taking $<200 \mu \mathrm{g} / \mathrm{d}^{(33)}$. High maternal folate status during pregnancy has also been associated with a $30 \%$ increase in the risk of childhood atopic dermatitis in a study of 8742 children, although there was no association with FA supplementation ${ }^{(34)}$. This suggests that FA rather than folate may be the important factor in determining the risk of allergic disease. However, other studies have not supported these findings. Martinussen et $a l^{(35)}$ found that FA supplementation during the first trimester of pregnancy in 1499 women had no association with asthma in the children at 6 years of age, which is consistent with the findings of Whitrow et $a l^{(32)}$ in 5-year-old children. Magdelijns et $a{ }^{(36)}$ also did not find a significant association between maternal FA supplementation and wheeze, lung function or asthma in 2834 children aged 6-7 years. Furthermore, there was no association in a study of 3786 children between maternal FA supplement use during the first trimester and asthma, wheeze, lower respiratory tract infections or eczema at 8 years, although there was a transient association with wheeze (prevalence ratio 1.20) at 12 months $^{(37)}$. Serum folate status at 2 years of age has been shown to be related inversely to total serum IgE concentration, atopy and wheeze in a study of 8083 children $^{(38)}$. Thus, subsequent folate intake may either modify or supersede the effect of maternal FA intake during early pregnancy on allergic outcomes. Together, these findings suggest that maternal FA supplementation may affect the risk of allergy in young children, but that this effect decreases in older children, possibly reflecting the maturation of the immune system.

Adult $F_{1}$ offspring of mice fed a diet with high FA content $(17 \mathrm{mg} / \mathrm{kg}$ feed) during pregnancy showed greater airway responsiveness to the muscarinic receptor agonist methacholine, which is a characteristic of patients with asthma, compared with the offspring of dams fed a diet with an adequate FA content $(2 \mathrm{mg} / \mathrm{kg} \text { feed })^{(39)}$. Airway challenge with nebulised ovalbumin induced a greater lung allergic response in the offspring of dams fed the high-FA diet than those of dams fed adequate FA. These effects were transmitted to the $\mathrm{F}_{2}$ generation via the male line, which implies changes in epigenetic marks in germ cells. Increased airway function responsiveness was associated with hypermethylation of the runt-related transcription factor-3 promoter that suggests a causal mechanism between maternal FA intake and impaired respiratory function ${ }^{(39)}$. However, the difference in FA intake between supplemented and control dams was substantially greater in this study than between women who do or do not take FA supplements during pregnancy. This may explain why the effects of FA were persistent in mice, but decreased with increasing time in human subjects. Nevertheless, these findings in mice support an effect of prenatal FA exposure on future respiratory function via a plausible epigenetic mechanism.

\section{Effect of maternal folic acid supplementation on metabolic outcomes in the offspring}

In two studies, the relationship between maternal FA intake and metabolic outcomes in children has been examined. No relationship was found between maternal FA intake and body composition in 5783 children aged 9 years ${ }^{(40)}$ or insulin resistance in 1132 children aged between 6 and 8 years, even in cobalamin-deficient mothers ${ }^{(41)}$. However, maternal erythrocyte folate status at 28 weeks in 700 women was associated positively with insulin resistance in children at 6 years, which the authors suggest reflected adherence to the recommendation that pregnant women in India take $500 \mu \mathrm{g}$ FA and $60 \mathrm{mg}$ Fe daily from 18 weeks of gestation to prevent anaemia, which was exacerbated by lower cobalamin status ${ }^{(42)}$. Hoyo et $a l^{(43)}$ found in a study of 438 women that methylation of the H19 differentially methylated region, which regulates insulin-like growth factor-2 expression, in cord blood leucocytes was reduced by $2.8 \%$ in 428 women who took FA supplements before conception and by $4.9 \%$ in 223 women who took FA supplements after conception, compared with those who did not take FA supplements, with a significant offspring sex difference, although phenotypic outcomes have not been reported. These findings support the suggestion that the effects of maternal FA supplementation on the phenotype of the offspring are mediated via epigenetic changes, but the precise nature of the effect may be influenced by the environmental context.

Despite the conflicting findings from human studies, investigations in animal models have shown a consistent effect of increased maternal FA intake on gene expression in the offspring. Moderate restriction of maternal protein intake in rodents has been shown to induce a number of phenotypic changes in the offspring ${ }^{(44)}$. Increasing FA intake by 5 -fold in dams fed a protein-restricted (PR) diet prevented altered promoter methylation and the expression of genes encoding hepatic PPAR $\alpha$ and the glucocorticoid receptor in the juvenile offspring by the PR diet alone ${ }^{(45)}$. However, sequence analysis of the PPAR $\alpha$ promoter in the liver of the offspring of dams fed the PR diet supplemented with FA showed hypermethylation of specific $\mathrm{CpG} \operatorname{loci}^{(46)}$. One possible implication is that some epigenetic changes induced in early life may be cryptic under normal physiological conditions, but may contribute to an altered phenotype when the organism is challenged by environmental stress. If so, this suggests a mechanism by 
which epigenetic changes may exert little short-term effect, but may contribute to future changes in phenotype such as altered health status. Furthermore, analysis of the liver transcriptome identified 175 genes which differed between the adult male offspring of dams fed a FA-supplemented diet during pregnancy and controls including the down-regulation of genes in the antioxidant ontology and the up-regulation of genes in the fatty acid metabolism ontology ${ }^{(47)}$. These findings provide direct evidence for induction by FA exposure during development of persistent changes in hepatic gene activity. Such effects could, for example by reducing antioxidant defence, induce vulnerabilities that are not pathogenic per se, but may contribute to increased disease risk. Hoile et al. ${ }^{(48)}$ found that maternal FA supplementation in rats induced altered methylation of specific CpG loci in the hepatic phosphoenolpyruvate carboxykinase promoter in adult female, but not male, rat offspring in a manner which differed directionally between CpG dinucleotides. Chmurzynska et al. ${ }^{(49)}$ found that a 5-fold increase in FA intake during pregnancy decreased body weight at 16 weeks of age, which was accompanied by significant effects on blood lipids and decreased expression of liver PPAR $\alpha$, PPAR $\gamma$ and the liver$\mathrm{X}$-receptor- $\alpha$, and lower hepatic phosphatidylethanolamine$\mathrm{N}$-methyltransferase, betaine-homocysteine methyltransferase ad cystathionine $\beta$-synthase ${ }^{(50)}$.

The effect of FA supplementation on the phenotype of the offspring appears to be modified by the overall nutrient composition of the diet. Feeding pregnant rats a PR diet induced increased systolic blood pressure, impaired acetylcholinemediated vasodilatation and reduced endothelial NO synthase mRNA expression ${ }^{(51)}$. These changes in vascular function were prevented by supplementation of the maternal PR diet with $5 \mathrm{mg} / \mathrm{kg}$ feed of FA. However, there was no effect of FA supplementation on vascular function in the offspring of dams fed a diet with adequate protein content ${ }^{(51)}$. In contrast, the offspring of rats fed a PR diet supplemented with FA also showed reduced post-weaning weight gain, and lower fasting plasma TAG, NEFA, $\beta$-hydroxybutyrate and glucose concentrations than the offspring of dams fed the PR diet containing adequate $\mathrm{FA}^{(52)}$. However, FA supplementation of the control, protein-sufficient diet induced increased fasting plasma lipid and glucose concentrations ${ }^{(52)}$. One implication of these findings is that that the effects of maternal FA supplementation on the offspring may differ between tissues to the extent of inducing opposing effects that are consistent with differences in the epigenome between cell types. This represents a potential major challenge for making nutritional recommendations to the general public and for the design of studies in human subjects because the relevant tissues may not be readily accessible and thus the effects of maternal FA supplementation may not be detected.

\section{Effect of maternal folic acid supplementation on cancer- related outcomes in the offspring}

Although FA fortification has been the focus of investigation with respect to colorectal cancer $^{(53)}$, there is limited information about the effect of maternal FA supplementation on cancer risk in the offspring. A case-control study of the relationship between maternal FA supplementation and the incidence of acute lympoblastic leukaemia in 393 children up to 14 years of age (1249 controls) found no significant effect of increased FA intake before or during pregnancy ${ }^{(54)}$ This finding was supported by a meta-analysis of similar studies, although vitamin supplementation, in general, was associated with a significant reduction in risk (OR 0.83). The authors conclude that any effect of multivitamin supplements on acute lympoblastic leukaemia is unlikely to be due to FA specifically.

Feeding rats a diet containing $5 \mathrm{mg} / \mathrm{kg}$ feed of FA before pregnancy and during pregnancy and lactation increased the rate of formation and the number of mammary tumours induced by 7,12-dimethylbenz[ $a$ ]anthracene and was associated with lower global DNA methylation in tumours from the adult offspring at 28 weeks of age compared with the offspring of dams fed a diet containing $2 \mathrm{mg} / \mathrm{kg}$ feed ${ }^{(55)}$. In contrast, FA supplementation during pregnancy and lactation in mice reduced the number of terminal end buds, the structure that gives rise to mammary tumours, which suggests a lower risk of mammary tumours ${ }^{(56)}$. One important difference between these studies is whether or not a tumour-inducing agent was used. Thus, differences in the effect of maternal FA supplementation on mammary cancer may reflect the latency period before tumour formation and/or the interactions between the methylome and the carcinogen. Using the same dietary design as Ly et al. ${ }^{(55)}$, maternal FA supplementation was also found to decrease the risk of azoxymethane-induced colorectal cancer by $64 \%$ in 31-week-old offspring ${ }^{(57)}$. These findings emphasise the potential for the tissue-specific effect of FA supplementation in humans.

\section{Effect of maternal folic acid supplementation on congenital heart defects}

In two studies, it has been investigated whether maternal periconceptional FA supplementation protects against congenital heart malformations. A case-control study of the effect of periconceptional multivitamin supplementation involving 958 cases and 3029 controls showed a $24 \%$ reduction in the risk of non-syndromic cardiac defects, a $54 \%$ reduction in the risk of outflow tract defects and a $39 \%$ reduction in ventricular septal defects ${ }^{(58)}$. A Hungarian randomised trial of periconceptional multivitamin supplementation also showed a $58 \%$ reduction in overall reduction in the risk of congenital heart defects ${ }^{(59)}$. Although both studies used supplements containing FA, neither was able to investigate the effects of individual components of the supplements. However, a recent case-control study designed to test the specific effect of FA supplementation showed an overall $18 \%$ reduction in congenital heart defects, with a $38 \%$ reduction in septal defects. Such beneficial effects may be the result of a direct effect of FA on the transcriptome of the developing heart ${ }^{(60)}$. However, FA supplementation did not reduce the risk of congenital defects in the offspring of diabetic mothers ${ }^{(61)}$. 


\section{Conclusions and research needs}

The findings of studies of human subjects have demonstrated the associations between maternal FA intake and phenotypic variation in children. These observations are complemented by the findings of studies in animal models that provide a mechanistic basis for these observations via the effects on the epigenome. Since the evaluation of FA toxicity did not include the assessment of epigenetic effects, one implication of these findings is that the assessment of FA safety may need to be revised to take into account epigenetic effects. Together, these findings raise important considerations about induction of adverse health effects in offspring by FA supplementation during pregnancy. First, what is the magnitude of the effect of FA intake during pregnancy on the phenotype of the offspring? There are currently too few data on which to base conclusions and the available estimate differs markedly between health outcomes. If the effect of maternal FA supplementation on the offspring is large, at least for some outcomes, to what extent is this acceptable when balanced against prevention of NTD? Although genetic background may exert an influence on the effect of prenatal FA exposure on health outcomes, because the effect of FA supplementation operates through epigenetic plasticity rather than genetic vulnerability, the potential number of individuals affected is likely to be greater than the number of NTD pregnancies. Again, this would need to be balanced against NTD prevention. The limited evidence reported to date precludes drawing firm conclusions, but is sufficient to raise concern. More studies are needed to address this, preferably randomised controlled trials, although these have ethical implications, into the effects of maternal FA intake on the epigenome and phenotype of the child across a wide age range to assess the stability of such changes in postnatal life. Such investigations need to be underpinned by information about the validity of proxy tissues that can be obtained readily in study cohorts, and experiments in model systems to provide mechanistic links between exposure to FA during development, and epigenetic and phenotypic outcomes. The potential for modulation of the effects of FA on epigenetic processes and phenotypic outcomes by other nutrients, by environmental, genetic and ethnic factors and by metabolic effects, such as capacity for DNA synthesis during development, need to be clarified. Answering these questions will involve considerable technical and financial challenges. However, in the face of the implementation of FA fortification in a number of countries and the ongoing debate about this strategy in others, failure to address the nature and extent of the effects of prenatal FA exposure on the offspring may have negative implications for future public health.

\section{Acknowledgements}

This work was not supported by a specific grant. G. C. B. wrote the first draft with substantial input from K. A. L. The authors declare no conflict of interest.

\section{References}

1. Taruscio D, Carbone P, Granata O, et al. (2011) Folic acid and primary prevention of birth defects. Biofactors 37 , $280-284$.

2. Blencowe H, Cousens S, Modell B, et al. (2010) Folic acid to reduce neonatal mortality from neural tube disorders. Int J Epidemiol 39, Suppl. 1, i110-i121.

3. Hibbard BM (1964) The role of folic acid in pregnancy; with particular reference to anaemia, abruption and abortion. J Obstet Gynaecol Br Commonw 71, 529-542.

4. Mulinare J, Cordero JF, Erickson JD, et al. (1988) Periconceptional use of multivitamins and the occurrence of neural tube defects. JAMA 260, 3141-3145.

5. Bower C \& Stanley FJ (1989) Dietary folate as a risk factor for neural-tube defects: evidence from a case-control study in Western Australia. Med J Aust 150, 613-619.

6. Werler MM, Shapiro S \& Mitchell AA (1993) Periconceptional folic acid exposure and risk of occurrent neural tube defects. JAMA 269, 1257-1261.

7. Shaw GM, Schaffer D, Velie EM, et al. (1995) Periconceptional vitamin use, dietary folate, and the occurrence of neural tube defects. Epidemiology 6, 219-226.

8. Smithells RW, Nevin NC, Seller MJ, et al. (1983) Further experience of vitamin supplementation for prevention of neural tube defect recurrences. Lancet i, 1027-1031.

9. Group MVSR (1991) Prevention of neural tube defects: results of the Medical Research Council Vitamin Study. Lancet 338, 131-137.

10. Laurence KM, James N, Miller MH, et al. (1981) Double-blind randomised controlled trial of folate treatment before conception to prevent recurrence of neural-tube defects. Br Med J (Clin Res Ed) 282, 1509-1511.

11. Kirke PN, Daly LE \& Elwood JH (1992) A randomised trial of low dose folic acid to prevent neural tube defects. The Irish Vitamin Study Group. Arch Dis Child 67, 1442-1446.

12. Vergel RG, Sanchez LR, Heredero BL, et al. (1990) Primary prevention of neural tube defects with folic acid supplementation: Cuban experience. Prenat Diagn 10, 149-152.

13. Scientific Advisory Committee on Nutrition (2006) Folate and Disease Prevention. London: TSO.

14. Finer LB \& Henshaw SK (2006) Disparities in rates of unintended pregnancy in the United States, 1994 and 2001. Perspect Sex Reprod Health 38, 90-96.

15. Ray JG, Singh G \& Burrows RF (2004) Evidence for suboptimal use of periconceptional folic acid supplements globally. BJOG 111, 399-408.

16. Crozier SR, Robinson SM, Borland SE, et al. (2009) Do women change their health behaviours in pregnancy? Findings from the Southampton Women's Survey. Paediatr Perinat Epidemiol 23, 446-453.

17. Crider KS, Bailey LB \& Berry RJ (2011) Folic acid food fortification - its history, effect, concerns, and future directions. Nutrients 3, 370-384.

18. Institute of Medicine (1998) Folaie. In Dietary Reference Intkaes for Thiamin, Riboflavin, Niacin, Vitamin $B_{6}$, Pantothenic Acid, Biotin and Choline, pp. 196-305. Washington, DC: National Academy Press.

19. Hunter R, Barnes J, Oakeley HF, et al. (1970) Toxicity of folic acid given in pharmacological doses to healthy volunteers. Lancet i, 61-63.

20. Hellstrom L (1971) Lack of toxicity of folic acid given in pharmacological doses to healthy volunteers. Lancet i, 59-61.

21. Richens A (1971) Toxicity of folic acid. Lancet $\mathbf{i}, 912$.

22. Sheehy TW (1973) Folic acid: lack of toxicity. Lancet $\mathbf{i}, 37$. 
23. Barres R, Yan J, Egan B, et al. (2012) Acute exercise remodels promoter methylation in human skeletal muscle. Cell Metab 15, 405-411.

24. Bird A (2002) DNA methylation patterns and epigenetic memory. Genes Develop 16, 6-21.

25. Fuks F, Burgers WA, Brehm A, et al. (2000) DNA methyltransferase Dnmt1 associates with histone deacetylase activity. Nat Genet 24, 88-91.

26. Rountree MR, Bachman KE \& Baylin SB (2000) DNMT1 binds HDAC2 and a new co-repressor, DMAP1, to form a complex at replication foci. Nat Genet 25, 269-277.

27. Fryer AA, Nafee TM, Ismail KM, et al. (2009) LINE-1 DNA methylation is inversely correlated with cord plasma homocysteine in man: a preliminary study. Epigenetics $\mathbf{4}$, 394-398.

28. Gluckman PD, Hanson MA, Cooper C, et al. (2008) Effect of in utero and early-life conditions on adult health and disease. $N$ Engl J Med 359, 61-73.

29. Wolff GL, Kodell RL, Moore SR, et al. (1998) Maternal epigenetics and methyl supplements affect agouti gene expression in Avy/a mice. FASEB J 12, 949-957.

30. Waterland RA, Dolinoy DC, Lin JR, et al. (2006) Maternal methyl supplements increase offspring DNA methylation at axin fused. Genesis 44, 401-406.

31. Haberg SE, London SJ, Stigum H, et al. (2009) Folic acid supplements in pregnancy and early childhood respiratory health. Arch Dis Child 94, 180-184.

32. Whitrow MJ, Moore VM, Rumbold AR, et al. (2009) Effect of supplemental folic acid in pregnancy on childhood asthma: a prospective birth cohort study. Am J Epidemiol 170, $1486-1493$.

33. Dunstan JA, West C, McCarthy S, et al. (2012) The relationship between maternal folate status in pregnancy, cord blood folate levels, and allergic outcomes in early childhood. Allergy 67, 50-57.

34. Kiefte-de Jong JC, Timmermans S, Jaddoe VWV, et al. (2012) High circulating folate and vitamin B-12 concentrations in women during pregnancy are associated with increased prevalence of atopic dermatitis in their offspring. $J$ Nutr 142, 731-738.

35. Martinussen MP, Risnes KR, Jacobsen GW, et al. (2012) Folic acid supplementation in early pregnancy and asthma in children aged 6 years. Am J Obstet Gynecol 206, 72e $71-72 \mathrm{e} 77$.

36. Magdelijns FJH, Mommers M, Penders J, et al. (2011) Folic acid use in pregnancy and the development of atopy, asthma, and lung function in childhood. Pediatrics 128, e135-e144

37. Bekkers MBM, Elstgeest LEM, Scholtens S, et al. (2012) Maternal use of folic acid supplements during pregnancy, and childhood respiratory health and atopy. Eur Respir J 39, 1468-1474.

38. Matsui EC \& Matsui W (2009) Higher serum folate levels are associated with a lower risk of atopy and wheeze. J Allergy Clin Immunol 123, 1253-1259.e2.

39. Hollingsworth JW, Maruoka S, Boon $\mathrm{K}$, et al. (2008) In utero supplementation with methyl donors enhances allergic airway disease in mice. $J$ Clin Invest 118, 3462-3469.

40. Lewis SJ, Leary S, Davey Smith G, et al. (2009) Body composition at age 9 years, maternal folate intake during pregnancy and methyltetrahydrofolate reductase (MTHFR) C677T genotype. Br J Nutr 102, 493-496.

41. Stewart CP, Christian P, Schulze KJ, et al. (2011) Low maternal vitamin B-12 status is associated with offspring insulin resistance regardless of antenatal micronutrient supplementation in rural Nepal. J Nutr 141, 1912-1917.
42. Yajnik CS, Deshpande SS, Jackson AA, et al. (2008) Vitamin $B(12)$ and folate concentrations during pregnancy and insulin resistance in the offspring: the Pune Maternal Nutrition Study. Diabetologia 51, 29-38.

43. Hoyo C, Murtha AP, Schildkraut JM, et al. (2011) Methylation variation at IGF2 differentially methylated regions and maternal folic acid use before and during pregnancy. Epigenetics 6, 928-936.

44. Bertram CE \& Hanson MA (2001) Animal models and programming of the metabolic syndrome. Br Med Bull $\mathbf{6 0}$, $103-121$

45. Lillycrop KA, Phillips ES, Jackson AA, et al. (2005) Dietary protein restriction of pregnant rats induces and folic acid supplementation prevents epigenetic modification of hepatic gene expression in the offspring. $J$ Nutr 135, $1382-1386$.

46. Lillycrop KA, Phillips ES, Torrens C, et al. (2008) Feeding pregnant rats a protein-restricted diet persistently alters the methylation of specific cytosines in the hepatic PPARalpha promoter of the offspring. Br J Nutr 100, 278-282.

47. Lillycrop KA, Rodford J, Garratt ES, et al. (2010) Maternal protein restriction with or without folic acid supplementation during pregnancy alters the hepatic transcriptome in adult male rats. BrJ Nutr 103, 1711-1719.

48. Hoile SP, Lillycrop KA, Grenfell LR, et al. (2012) Increasing the folic acid content of maternal or post-weaning diets induces differential changes in phosphoenolpyruvate carboxykinase mRNA expression and promoter methylation in rats. Br J Nutr 108, 852-857.

49. Chmurzynska A, Stachowiak M, Gawecki J, et al. (2012) Protein and folic acid content in the maternal diet determine lipid metabolism and response to high-fat feeding in rat progeny in an age-dependent manner. Genes Nutr $\mathbf{7}$, $223-234$

50. Chmurzynska A \& Malinowska AM (2011) Homocysteine homeostasis in the rat is maintained by compensatory changes in cystathionine beta-synthase, betaine-homocysteine methyltransferase, and phosphatidylethanolamine $\mathrm{N}$-methyltransferase gene transcription occurring in response to maternal protein and folic acid intake during pregnancy and fat intake after weaning. Nutr Res 31, 572-578.

51. Torrens C, Brawley L, Anthony FW, et al. (2006) Folate supplementation during pregnancy improves offspring cardiovascular dysfunction induced by protein restriction. Hypertension 47, 982-987.

52. Burdge GC, Lillycrop KA, Jackson AA, et al. (2008) The nature of the growth pattern and of the metabolic response to fasting in the rat are dependent upon the dietary protein and folic acid intakes of their pregnant dams and post-weaning fat consumption. Br J Nutr 99, 540-549.

53. Mason JB, Dickstein A, Jacques PF, et al. (2007) A temporal association between folic acid fortification and an increase in colorectal cancer rates may be illuminating important biological principles: a hypothesis. Cancer Epidemiol Biomarkers Prev 16, 1325-1329.

54. Milne E, Royle JA, Miller M, et al. (2010) Maternal folate and other vitamin supplementation during pregnancy and risk of acute lymphoblastic leukemia in the offspring. Int J Cancer 126, 2690-2699.

55. Ly A, Lee H, Chen J, et al. (2011) Effect of maternal and postweaning folic acid supplementation on mammary tumor risk in the offspring. Cancer Res 71, 988-997.

56. Sie KKY, Chen J, Sohn K-J, et al. (2009) Folic acid supplementation provided in utero and during lactation reduces the number of terminal end buds of the developing mammary glands in the offspring. Cancer Lett 280, 72-77. 
57. Sie KKY, Medline A, van Weel J, et al. (2011) Effect of maternal and postweaning folic acid supplementation on colorectal cancer risk in the offspring. Gut 60, 1687-1694.

58. Botto LD, Mulinare J \& Erickson JD (2000) Occurrence of congenital heart defects in relation to maternal mulitivitamin use. Am J Epidemiol 151, 878-884.

59. Czeizel AE (1998) Periconceptional folic acid containing multivitamin supplementation. Eur J Obstet Gynecol Reprod Biol 78, 151-161.
60. Caldwell PT, Manziello A, Howard J, et al. (2010) Gene expression profiling in the fetal cardiac tissue after folate and low-dose trichloroethylene exposure. Birth Defects Res A Clin Mol Teratol 88, 111-127.

61. Banhidy F, Dakhlaoui A, Puho EH, et al. (2011) Is there a reduction of congenital abnormalities in the offspring of diabetic pregnant women after folic acid supplementation? A population-based case-control study. Congenit Anom 51, $80-86$. 